G. Kuhn

Nagoya Math. J.

Vol. 128 (1992), 95-120

\title{
ANISOTROPIC RANDOM WALKS ON FREE PRODUCTS OF CYCLIC GROUPS, IRREDUCIBLE REPRESENTATIONS AND IDEMPOTENTS OF $C_{\mathrm{reg}}^{*}(G)$
}

\author{
GABRIELLA KUHN
}

\section{Introduction}

Let $G=\mathbf{Z}_{n+1} * \mathbf{Z}_{n+1} * \cdots * \mathbf{Z}_{n+1}=\left\langle a_{1}, a_{2}, \ldots, a_{q+1} \mid a_{j}^{n+1}=e \forall j\right\rangle$ be the free product of $q+1$ copies of $\mathbf{Z}_{n+1}$, and let $\mathscr{G}$ denote its Cayley graph (with respect to $a_{j}, 1 \leq j \leq q+1$ ). We may think of $G$ as a group acting on the "homogeneous space" $\mathscr{G}$, This point of view is inspired by the case of $S L_{2}(\mathbf{R})$ acting on the hyperbolic disk and is developed in [FT-P] [I-P] [FT-S] [S] (but see also $[\mathrm{C}])$.

Since $G$ is a group we may investigate some classical topics: the full (reductive) $C^{*}$ algebra, its dual space, the regular Von Neumann algebra and so on. See $[B][P][L] ~[V]$ and also [H]. These approaches give results pointing up the analogy between harmonic analysis on these groups and harmonic analysis on more classical objects.

On the other hand, since $G$ is not type $\mathbf{I}$, its representation theory cannot be completely analogous to that of $S L_{2}(\mathbf{R})$ and indeed will never be completely under control. Nevertheless, the probabilistic approach, as in [FT-S] and [S] provides an important instrument of investigation.

In order to understand this, recall that $\mathscr{G}$ is a connected graph with the property that at each vertex $V$ there meet exactly $q+1$ polygons $\mathscr{P}_{j}\left(V_{j}\right)(j=1, \ldots$, $q+1$ ) each with $n+1$ sides. Identify the vertices of $\mathscr{G}$ with the elements of $G$. Then, if $V$ corresponds to $g \in G$, the polygon $\mathscr{P}_{j}(V)$ is given by the coset $g\left(\mathbf{Z}_{n+1}\right)_{j}$ where $\left(\mathbf{Z}_{n+1}\right)_{j}$ is the $j$ 'th copy of $\mathbf{Z}_{n+1}$ in the free product expression for $G$.

Choose $q+1$ positive numbers $p_{q+1} \leq p_{q} \leq \cdots \leq p_{1}$ with $\sum_{j=1}^{q+1} p_{j}=1$.

Consider the random walk where the transition probability $p\left(V^{\prime} \rightarrow V\right)$ of moving from a vertex $V^{\prime}$ to a vertex $V$ is $p_{j} / n$ if $V^{\prime} \in \mathscr{P}_{j}(V)$ and $V^{\prime} \neq V$. Fix the

Received October 11, 1989. 
vertex $V_{0}$ corresponding to the identity $e$ of $G$ and let $\mu(x)=p\left(V_{0} \rightarrow x \cdot V_{0}\right)$ where $x \cdot V_{0}$ denotes the element of $\mathscr{G}$ corresponding to $x$. Consider the (bounded) operator $M$ from $l^{2}(G)$ to $l^{2}(G)$ given by right convolution with $\mu$. We know that we can realize the spectral decomposition of $M$ using the Green function associated to the above random walk.

This technique is carried out in [I-P] (with $\left.p_{1}=p_{2}=\cdots=p_{q+1}=\frac{1}{q+1}\right)$ and in [S] (with $n+1=2$ and $p_{q+1} \leq p_{q} \leq \cdots \leq p_{1}$ ) to obtain a decomposition of the left regular representation into a direct integral of representations acting on (generalized) eigenspaces of $\mu$. The decomposition is into irreducibles exactly when there are no true eigenspaces of $\mu$. We know that the decomposition is into irreducibles in the case $n+1=2$.

The aim of this paper is to investigate the case $n+1>2$. In this case the shape of the spectrum of $M$ depends on the pair $(q, n)$ as well as on the choice of the $p_{j}$. (In contrast with the case $n+1=2$, the dependence on the $p_{j}$ is quite complicated). The discrete part of the spectrum corresponds to eigenspaces of $\mu$, and hence to $G$-invariant subspaces of $l^{2}(G)$. Such subspaces are always reducible representations (see [C-FT]).

The continuous part always gives rise to irreducible representations in generalized eigenspaces. We shall see that such a discrete part occurs if and only if $q<n$ (independently of the $p_{j}$ ). We shall also see that, if the $p_{j}$ are close enough, the continuous spectrum consists only of one interval, but, if the $p_{j}$ are far enough apart, then the continuous spectrum splits into many pieces. Since every connected component of the spectrum defines a nontrivial idempotent in $C_{\mathrm{reg}}^{*}(G)$, we cannot get an arbitrary number of pieces. We shall see that it is possible to choose the $p_{j}$ in such a way that the number of pieces is the largest possible, consistent with the $K$-theory.

The resolvent of $M$ has also been considered in $[\mathrm{C}-\mathrm{S}]$ and $\left[\mathrm{T}_{2}\right]$. In particular the case $q+1=2$ is completely described in the above two papers.

Here we shall give a complete description of the spectrum of $\mu$ in several cases. Our main reference is $[\mathrm{S}]$ and the reader should refer to it in order to verify the theorems which are not proved here (see Section 4). The final version of this paper was written while the author was visiting the University of Chicago.

In particular we thank Tim Steger who spent a great amount of time in helpful conversations; providing suggestions, examples and references on the subject of this paper. 


\section{$\S 1$. Notation}

Let $G=\left\langle a_{1}, a_{2}, \ldots, a_{q+1} \mid a_{j}^{n+1}=e \forall j\right\rangle$. Each $x$ in $G, x \neq e$, may be written uniquely, as a reduced word, as $x=a_{j_{1}}^{h_{1}} a_{j_{2}}^{h_{2}} \cdots a_{j m}^{h_{m}}$ where $1 \leq h_{l} \leq n$ for each $l$. The Cayley graph of $G$ with respect to the generators $\left(a_{j}\right)_{j=1}^{q+1}$ is the graph $\mathscr{G}$ described in the introduction. The Cayley graph, $\mathscr{G}^{\prime}$, of $\mathscr{G}$, with respect to the generators $\left\{a_{j}^{k}\right\}_{j=1}^{q+1} \quad n_{k=1}^{n}$ is more closely adapted to this problem.

One obtain $\mathscr{G}^{\prime}$ from $\mathscr{G}$ by connecting two vertices whenever they belong to a common polygon. The group $G$ acts on $\mathscr{G}$ (or on $\mathscr{G}^{\prime}$ ) by left multiplication. The distance between any two vertices $V_{1}$ and $V_{2}$ of $\mathscr{G}$, is defined as the minimum number of polygons you must cross in order to go from $V_{1}$ to $V_{2}$. The corresponding length on $G$ is the block length, given by $|x|=m$ when $a_{j_{1}}^{h_{1}} a_{j_{2}}^{h_{2}} \cdots a_{j_{m}}^{h m}$ is the reduced word for $x$. Then $\operatorname{dist}(x, y)=\left|y^{-1} x\right|$. Observe that this distance on $\mathscr{G}$ is ordin. ary path length on $\mathscr{G}^{\prime}$. For each $j(j=1, \ldots, q+1)$ consider the probability measure $\mu$, which is equidistributed on the set $\left(\mathbf{Z}_{n+1}\right)_{j} \backslash\{e\}$. Let $\mu$ be a convex linear combination of $\mu_{j}$, so that

$$
\mu(x)=\sum_{j=1}^{q+1} p_{j} \mu_{j}(x)=\sum_{j=1}^{q+1} \sum_{k=1}^{n} \frac{p_{1}}{n} \delta_{a_{j}^{*}}
$$

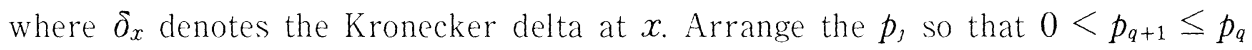
$\leq \cdots \leq p_{1}$. The transition matrix $\{R(x, y)\}$ of the random walk described in the introduction is defined by the law $R(x, y)=\operatorname{prob}(x \rightarrow y)=\mu\left(x^{-1} y\right)$. Let $M$ : $l^{2}(G) \rightarrow l^{2}(G)$ be the operator of right convolution with $\mu$. Since $\mu$ commutes with left convolution, we know that the spectral decomposition of $M$ leads to a decomposition of the left regular representation of $G$. Let us denote by $\operatorname{sp}(\mu)$ (respec tively $\operatorname{res}(\mu)$ ) the spectrum (respectively the resolvent) of the operator $M$. The functional calculus says that

$$
\delta_{e}(x)=\frac{1}{2 \pi i} \int_{C}(\gamma-\mu)^{-1}(x) d \gamma
$$

where $C$ is a smooth curve containing $\operatorname{sp}(\mu)$ inside and, letting $C$ shrink around the spectrum of $\mu$.

$$
\delta_{e}(x)=\frac{1}{\pi} \int_{\mathrm{sp}(\mu)} \operatorname{Im} g_{\sigma}(x) d \sigma+\sum_{j=1}^{N} P_{j}(x)
$$

where

$$
\operatorname{Im} g_{\sigma}(x)=\lim _{\varepsilon \rightarrow 0^{+}}\left\{(\sigma+i \varepsilon-\mu)^{-1}(x)-(\sigma-i \varepsilon-\mu)^{-1}(x)\right\}
$$

and $p_{1}(x)$ are mutually orthogonal projections into the $l^{2}$ eigenspaces of $\mu$ (corres- 
ponding to the poles of the analytic function $\left.(\gamma-\mu)^{-1}(x)\right)$. We shall denote by $d m(\sigma)$ the positive measure obtained by letting $x=e$ in 1.2. Hence

$$
d m(\sigma)=-\frac{1}{\pi} \operatorname{Im} g_{\sigma}(e) d \sigma+\sum_{j=1}^{N}\left\{\underset{\gamma=\gamma,}{\operatorname{Res}}(\gamma-\mu)^{-1}(e)\right\} \delta_{\gamma,}
$$

We shall see in Section 4 that, for almost all $\sigma,-\frac{1}{\pi} \operatorname{Im} g_{\sigma}(x)$ is a positive definite two sided eigenfunction of $\mu$. Hence we may use the Gelfand-Raikov construction to decompose $l^{2}(G)$ into generalized eigenspaces of $\mu$.

The next section is devoted to the computation of $(\gamma-\mu)^{-1}$.

\section{$\S 2$. Computation of $(\gamma-\mu)^{-1}$.}

Our goal is to describe $(\gamma-\mu)^{-1}(x)=g_{r}(x)$ in terms of $q+2$ complex functions of $\gamma$. It is well known (see [A-K], [C-S], [M-L], [S], [W], [V]) that $g_{\gamma}(x)$ may be written as a scalar multiple of function $h_{r}(x)$ satisfying

$$
\begin{aligned}
& h_{\gamma}(e)=1 \\
& h_{\gamma}(x y)=h_{\gamma}(x), h_{\gamma}(y) \quad \text { whenever }|x y|=|x|+|y| .
\end{aligned}
$$

As pointed out in $[\mathrm{S}]$, the above description depends, basically, on the following property of the graph $\mathscr{G}$ : any path, in $\mathscr{G}$, from $e$ to $a_{\jmath_{1}}^{h_{1}} a_{\jmath_{2}}^{h_{2}} \cdots a_{\jmath_{m}}^{h_{m}}$ must pass through $a_{j_{1}}^{h_{1}}$. Let us recall the method. An $m$-path $P$ from $x$ to $y$ is a sequence of $(m+1)$ elements of $G, P=\left(x_{m}, x_{m-1}, \ldots, x_{0}\right) \in G^{m+1}$, such that $x_{0}=e$ and $x_{m}=y$. An $m$-path stops at $y$ if $x_{j} \neq y$ for $0 \leq j \leq m-1$ and $x_{m}=y$. We denote by $\pi_{m}(y, x)$ the set of all $m$ paths from $x$ to $y$ and by $\pi_{m}(y, x,\{y\})$ the subset of $\pi_{m}(y, x)$ consisting of all $m$-paths which stop at $y$. Define the evaluation of a function $f$ along a path $P=\left(x_{m} \ldots x_{0}\right)$ to be $E(P, f)=$ $\Pi_{j=0}^{m-1} f\left(x_{j}^{-1} x_{j+1}\right)$. Set also

$$
E_{m}(y, x, \mu)=\sum_{P \in \pi m(y, x)} E(P, \mu)
$$

where the above sum is extended to all possible $m$-paths $P$ from $x$ to $y$. It is well known that

$$
(\gamma-\mu)^{-1}(x)=\gamma^{-1}\left[\sum_{m=0}^{\infty} E_{m}(x, e, \mu / \gamma)\right] .
$$

Suppose now that $x=a_{j_{1}}^{h_{1}} a_{j_{2}}^{h_{2}} \cdots a_{j_{s}}^{h_{s}}$, so that the first syllable of $x$ is $a_{j_{1}}^{h_{1}}$. If $P=$ $\left(x_{m}, \ldots, x_{0}\right)$ is any $m$-path from $e$ to $x$, then $a_{j_{1}}^{h_{1}}$ occurs at least once in the sequence $x_{m} \ldots x_{0}$. We may therefore think of $P$ as a "product" of two paths, say $P_{1}$ and $P_{2}$, where $P_{1}$ stops at $a_{j_{1}}^{h_{1}}$ and $P_{2}$ starts at $a_{j_{1}}^{h_{1}}$. Since $P_{1}$ stops at $a_{j_{1}}^{h_{1}}$ this decomposition is unique. We recall that our random walk is invariant with respect 
to the $G$ action on $\mathscr{G}$, namely we have:

$$
p(x \rightarrow y)=p(g x \rightarrow g y) \quad \forall x, y, g \in G
$$

Repeated applications of the above argument allow us to conclude that

$$
(\gamma-\mu)^{-1}(x)=\frac{1}{2 w(\gamma, \mu)} \cdot h_{\gamma}(x)=\frac{1}{2 w} h_{\gamma}(x)
$$

where $\frac{1}{2 w}=(\gamma-\mu)^{-1}(e), h_{\gamma}(x)$ satisfies 2.1 and

$$
h_{\gamma}\left(a_{j}^{k}\right)=E\left(\Pi\left(a_{j}^{k}, e ;\left\{a_{\jmath}^{k}\right\} ; \mu / \gamma\right)\right.
$$

where the right hand side of 2.4 means the evaluation of $\mu / \gamma$ along all paths from $e$ to $a_{j}^{k}$ which stops at $a_{j}^{k}$. We obseve that the probability of reaching a point $V=$ $a_{j_{1}}^{h_{1}} a_{j_{2}}^{h_{2}} \cdots a_{j_{N}}^{h_{N}}$ starting from $V_{0}=e$ depends only on the polygons you cross and not on the particular point you choose in every polygon, hence $\mu^{* m}\left(a_{11}^{h_{1}} a_{j_{2}}^{h_{2}} \ldots\right.$ $a_{j N}^{h_{N}}$ ) does not depend on the choice of the $N$-ple $\left(h_{1}, \ldots, h_{N}\right)$ (this fact can also be easily proved by induction). Observe that, for large values of $\gamma$, say $\gamma>1$, one has

$$
h_{\gamma}\left(a_{j}^{k}\right)=(2 w) \cdot(\gamma-\mu)^{-1}\left(a_{j}^{k}\right)=\sum_{m=0}^{\infty} \frac{\mu^{* m}\left(a_{j}^{k}\right)}{\gamma^{m}} .
$$

By analytic continuation, we can see that the quantities $h_{\gamma}\left(a_{j}^{k}\right)(k=1, \ldots, n)$ do not depend on $k$. Let us denote by $\xi_{j}=\xi_{j}(\gamma)$, their common value. Recalling that $g_{\gamma} *(\gamma-\mu)=(\gamma-\mu) * g_{\gamma}=\delta_{e}$ we can get the relations between $\gamma, w$ and the $\xi_{j}$ :

$$
\begin{aligned}
& \text { i) } \quad \gamma=2 w+\sum_{j=1}^{q+1} p_{j} \xi_{j} \\
& \text { ii) } \quad p_{j}\left(\xi_{j}-\frac{1}{n} \xi_{j}^{-1}\right)=\left(\frac{n-1}{n}\right) p_{j}-2 w \\
& \text { iii) } \quad \xi_{\jmath}=\frac{ \pm \sqrt{z_{j}^{2}+\frac{4 p_{j}^{2}}{n}-z_{j}}}{2 p_{\imath}} \text { where } z_{\jmath}=2 w-p_{\jmath}\left(\frac{n-1}{n}\right)
\end{aligned}
$$

Again, by analytic continuation, we may think that 2.3 and 2.5 hold for every $\gamma$ in $C \backslash \operatorname{sp}(\mu)$ that satisfy the condition $g_{\gamma}(e) \neq 0$. We want to investigate now the case $g_{\gamma}(e)=0$. We first need to estimate the $l^{2}$ norm of $g_{\gamma}(x)$. The estimation is essentially given in [S].

Lema 2.1. Let $h: G \rightarrow \mathbf{C}$ satisty 2.1. Suppose also that $h\left(a_{j}^{k}\right)=h\left(a_{j}\right)$ is independent of $k(1 \leq k \leq n)$. Then $h$ belongs to $l^{p}(G)$ if and only if 


$$
\sum_{j=1}^{q+1} \frac{n\left|h\left(a_{\jmath}\right)\right|^{p}}{1+n\left|h\left(a_{j}\right)\right|^{p}}<1
$$

Moreover, one has

$$
\|h\|_{p}^{p}=\left\{1-\sum_{j=1}^{q+1} \frac{n\left|h\left(a_{j}\right)\right|^{p}}{1+n\left|h\left(a_{j}\right)\right|^{p}}\right\}^{-1} .
$$

Proof. The proof is based on the fact that the same statements are true when $n=1$ (see [S]). For $1 \leq k \leq n$, let $h\left(a_{j}^{k}\right)=h_{j}$ and let $\tilde{h}(x)$ be the function defined on $Z_{2} * Z_{2} * \cdots * Z_{2}=\left\langle A_{1}, A_{2}, \ldots, A_{q+1} \mid A_{j}^{2}=e \forall j\right\rangle$ by the condition

$$
\tilde{h}\left(A_{i 1} A_{i 2} \cdots A_{i j}\right)=h_{i_{1}} h_{i_{2}} \cdots A_{i j}, \tilde{h}(e)=1 .
$$

For every $x$ in $G$, with $x=a_{t_{1}}^{k_{1}} a_{t_{2}}^{k_{2}} \cdots a_{i j}^{k j}$, let $\tilde{x}$ be the element of $Z_{2} * Z_{2} * \cdots *$ $Z_{2}$ defined by letting $\tilde{x}=A_{i_{1}} A_{i_{2}} \cdots A_{i j}$. Then

$$
\sum_{|x|=k}|h(x)|^{p}=n^{k} \sum_{|\tilde{x}|=k}|\tilde{h}(\tilde{x})|^{p}
$$

where the length of $\tilde{x}$ in the right hand side of the above equality is the block length in $Z_{2} * Z_{2} * \cdots * Z_{2}$.

Hence the $p$-norm of $h$ can be regarded as the $p$-norm of the function $(\sqrt[p]{n})^{|x|}$. $\tilde{h}(x)$ defined on $Z_{2} * Z_{2} * \cdots * Z_{2}$ and the theorem follows since the same formulas are true for $n=1$.

Remark. Formula 2.7 also appears in $\left[\mathrm{T}_{2}\right]$. Since our proof is very short we decided to include it, for the reader's convenience.

Suppose now that $g_{\gamma_{0}}(e)=0$. Then the function $w(\gamma)$ has a pole at $\gamma=\gamma_{0}$. Because of 2.5 i) at least one of the $\xi_{j}(\gamma)$ must have pole too. Since, for complex $\gamma, g_{\gamma}$ belongs to $l^{2}(G)$, one can see that exactly one of the $\xi_{j}$, say $\xi_{k}$, has a pole at $\gamma=\gamma_{0}$. Suppose that $k \neq 1$. Then

$$
\lim _{w \rightarrow+\infty}\left|\xi_{k} \xi_{1}\right|=\lim _{w \rightarrow \infty} \frac{p_{1}}{n p_{k}} \frac{\sqrt{z_{k}^{2}+4 \frac{p_{k}^{2}}{n}}+z_{k}}{\sqrt{z_{1}^{2}+4 \frac{p_{1}^{2}}{n}}+z_{1}}=\frac{p_{1}}{n p_{k}} .
$$

Let $\tilde{x}=a_{1}^{j_{1}} a_{k}^{h_{1}} a_{1}^{j_{2}} a_{k}^{h_{2}} \cdots a_{1}^{j_{s}} a_{k}^{h_{s}}$ with $|\tilde{x}|=2 s$. Then 2.8 implies that, for every element $\tilde{x}$ of this form, $\sum_{s=1}^{\infty} \sum_{|\tilde{x}|=2 s}\left|g_{j}(\tilde{x})\right|^{2}=+\infty$ in a neighbourhood of $\gamma=$ $\gamma_{0}$, which is a contradiction. So that the only possibility is that $\xi_{1}$ has a pole at $\gamma_{0}$. But $g_{r} *(\gamma-\mu)\left(a_{1}\right)=0$ implies

$$
\gamma-\left(\frac{n-1}{n}\right) p_{1}=\frac{p_{1}}{n} \cdot \frac{1}{\xi_{1}}+\sum_{j=2}^{q+1} p_{j} \xi_{j}
$$


Again since $g_{\gamma}$ belongs to $l^{2}(G)$ for complex $\gamma$, we see that, letting $\gamma \rightarrow \gamma_{0}$, the quantity $\left|\xi_{1} \xi_{1}\right|(j \neq 1)$ must be bounded. Since $\left|\xi_{1}\right| \rightarrow+\infty 2.9$ implies that $\gamma_{0}=\frac{n-1}{n} \cdot p_{1}$. A limit argument also shows that, if $g_{\bar{r}}(e)=0$ for some $\bar{\gamma}$ in $\operatorname{res}(\mu)$, then

$g_{\vec{r}}(x)=\left\{\begin{array}{cc}-\frac{1}{p_{1}} \cdot \prod_{\jmath=1}^{s}\left(\frac{-p_{i_{j}}}{n p_{1}}\right) \text { if } x=a_{1}^{k_{1}}\left(a_{i_{1}}^{h_{1}} a_{1}^{k_{2}}\right)\left(a_{t_{2}}^{h_{2}} a_{1}^{k_{3}}\right) \cdots\left(a_{l_{s}}^{h_{s}} a_{1}^{k_{s+1}}\right) \\ & \text { with }|x|=2_{s}+1 \\ 0 & \text { otherwise. }\end{array}\right.$

Since, for such $\bar{\gamma}$, we have

$$
\left\|g_{\gamma}\right\|_{2}^{2}=\frac{n}{p_{1}^{2}} \sum_{s=0}^{\infty} n^{2 s}\left(\sum_{j=2}^{q+1} \frac{p_{j}^{2}}{n^{2} p_{1}^{2}}\right)^{s}=\frac{n}{p_{1}^{2}} \sum_{s=0}^{\infty}\left(\sum_{j=2}^{q+1} \frac{p_{j}^{2}}{p_{1}^{2}}\right)^{s}
$$

we see that $\bar{\gamma} \in \operatorname{res}(\mu)$ and $g_{\bar{\gamma}}(e)=0$, implies

$$
p_{1}^{2}>\sum_{j=2}^{q+1} p_{j}^{2}
$$

On the other hand, a quick check shows that, when $p_{1}^{2}>\sum_{j=2}^{q+1} p_{j}^{2}$, the function defined by 2.10 satisfies the equation $\left(p_{1}\left(\frac{n-1}{n}\right)-\mu\right) * g_{\bar{\gamma}}=\delta_{e}$ so that $\bar{\gamma}=$ $p_{1}\left(\frac{n-1}{n}\right)$ belongs to res $(\mu)$. We may summarize with the following

LeNma 2.2. Let $\gamma$ be a complex number in $\operatorname{res}(\mu)$. If $g_{\gamma}(e) \neq 0$ then there exists a choice of signs in the equation $2.5 \mathrm{iii})$ such that $g_{\gamma}(x)$ has the form given in 2.3 with $h_{\gamma}$ satisfying 2.1 and $2.4, \gamma$ and $\xi$, as in 2.5 .

If $g_{\gamma}(e)=0$ then $\gamma=p_{1}\left(\frac{n-1}{n}\right)$ and $p_{1}^{2}>\sum_{j=2}^{q+1} p_{j}^{2}$.

Moreover, if $p_{1}^{2}>\sum_{j=2}^{q+1} p_{j}^{2}$ then $\gamma=p_{1}\left(\frac{n-1}{n}\right)$ belongs to res $(\mu)$ and $g_{\gamma}(e)=0$.

In order to give a general description of $\operatorname{sp}(\mu)$ we recall a fundamental relation beween the $l^{2}$ norm of $g_{\gamma}$ and the functions $\gamma(w)$ described in 2.5 i).

Suppose that $\gamma$ and $\gamma^{\prime}$ are large and real and let $\xi_{j}^{\prime}=\xi_{j}\left(\gamma^{\prime}\right), \xi_{j}=\xi_{j}(\gamma)$, $g_{\gamma}(e)=w, g_{\gamma^{\prime}}(e)=w^{\prime}$. Then, arguing as in [S], we get

$$
\frac{1}{2} \frac{\gamma^{\prime}-\gamma}{w^{\prime}-w}=1-\sum_{j=1}^{q+1} \frac{n \xi_{j} \xi_{j}^{\prime}}{1+n \xi_{j} \xi_{j}^{\prime}}
$$

and, letting $w^{\prime} \rightarrow w$, we hav 


$$
\frac{1}{2} \frac{d \gamma}{d w}=1-\sum_{1}^{q+1} \frac{n \xi_{j}^{2}}{1+n \xi_{j}^{2}}
$$

In particular, if $\gamma \in \mathbf{R} \backslash\left\{\operatorname{sp}(\mu) \cup\left\{p_{1}\left(\frac{n-1}{n}\right)\right\}\right\}$, there exists a choice of signs in 2.5 iii) such that $\gamma$ can be expressed as a function of $w$ according with $2.5 \mathrm{i}$ ). Moreover, since for such a $\gamma g_{\gamma}(e)$ is real, we have

$$
\frac{1}{2} \frac{d \gamma}{d w}=1-\sum_{j=1}^{q+1} \frac{n\left|\xi_{j}\right|^{2}}{1+n\left|\xi_{j}\right|^{2}}=\left\|g_{\gamma}\right\|_{2}^{2} .
$$

We may summarize with the following

THEOREM 2.1. Let $\gamma_{0} \in \mathbf{R} \backslash\left\{\operatorname{sp}(\mu) \cup\left\{p_{1}\left(\frac{n-1}{n}\right)\right\}\right\}$. Then there exists a choice of signs for the functions $\xi_{j}$ in 2.5 iii) such that $\gamma$ can be expressed as a function of $g_{\gamma}(e)=\frac{1}{2 w}$ according with $\left.2.5 \mathrm{i}\right)$. Moreover we have $\left.\frac{d \gamma}{d w}\right|_{w_{0}}>0$ where $2 w_{0}=$ $\frac{1}{g_{\gamma_{0}}(e)}$. Conversely, suppose there exists a choice of signs in 2.5 iii) and a $w_{0} \in R \backslash\{0\}$ such that the function $\gamma=\gamma(w)$ of 2.5 i) has positive derivative at $w=w_{0}$, then $\gamma_{0}=\gamma\left(w_{0}\right)$ belongs to res $(\mu)$.

$$
\begin{aligned}
& \text { If } p_{1}^{2}>\sum_{2}^{q+1} p_{j}^{2} \text { then } \gamma=p_{1}\left(\frac{n-1}{n}\right) \text { belongs to res }(\mu) \text {. } \\
& \text { If } p_{1}^{2} \leq \sum_{2}^{q+1} p_{j}^{2} \text { then, for } \gamma=p_{1}\left(\frac{n-1}{n}\right), g_{\gamma}(e) \text { cannot be zero, so that } p_{1}\left(\frac{n-1}{n}\right)
\end{aligned}
$$
belongs to res $(\mu)$ iff there exists a choice of signs in 2.5 iii) such that the correspon ding $\gamma(w)$ has positive derivative at $w_{1}$, with $\gamma\left(w_{1}\right)=p_{1}\left(\frac{n-1}{n}\right)$.

We conclude this section with the description of the discrete part of $\operatorname{sp}(\mu)$. The isolated points of $\operatorname{sp}(\mu)$ correspond to the poles of $g_{\gamma}(e)$, that is, to the zeros of $w=w(\gamma)$. Since the case $q=1$ has been completely described in [C-S] (see also $\left[\mathrm{T}_{2}\right]$ ) we shall confine our attention to $q>1$. Suppose that $w=0$. Since for complex values of $\gamma=\gamma(w) g_{\gamma}(x)$ belongs to $l^{2}(G)$ we must have

$$
1-\sum_{1}^{q+1} \frac{n\left|\xi_{j}(0)\right|^{2}}{1+n\left|\xi_{j}(0)^{\prime}\right|^{2}} \geq 0 \text {. }
$$

Now $\left|\xi_{\jmath}(0)\right|=1$ or $\frac{1}{n}$ according with the choice “+” or "-_" in 2.5 iii).

Suppose that $r$ of the $\xi_{1}$ have been chosen with the sign + and $(q+1-r)$ with the sign - . Since $\frac{2 n}{1+n}>1$ in our case, we can see that no more than 1 sign 
" $+"$ is allowed for the $\xi_{j}$. On the other hand, for $r=1$, we have $1-\frac{n}{1+n}$ $-\frac{q}{n+1} \geq 0$ again, since we are dealing with the case $q>1$ this is impossible.

So that all the signs in 2.5 iii) must be “-”. Again, we see that 2.14 is possible only if $q \leq n$. In particular, when $q>n, g_{r}(x)$ has no poles. Suppose now $q<n$.

Let $\mathcal{N}$ consist of all complex valued functions $f$, defined on $\mathscr{G}$, which have zero average over each polygon. It is easy to verify that $\mathcal{N}$ is an eigenspace for the operator induced on $\mathscr{G}$ by right convolution with $\mu$. If $f$ is such a function we have $f * \mu=-\frac{1}{n} f$. Of course we identify functions defined on $\mathscr{G}$ with functions defined on $G$.

Let $\mathcal{N}_{2}=\mathcal{N} \cap l^{2}(G)$. Then $\mathcal{N}_{2} \neq\{0\}$ if and only if $q<n$. This fact can be deduced by the behaviour of an isotropic random walk on $G$ and can also be deduced by $\left[T_{1}\right]$. For the reader's convenience we shall give a simple proof.

Suppose that $f \in \mathcal{N}_{2}$ and $f \not \equiv 0$. We may always suppose that $f(e) \neq 0$. We may take the average of $f$ on each set $E_{k}=\{x:|\dot{x}|=k\}$ to obtain a new function $\varphi_{f}$, which is constant on $E_{k}$.

By Swartz inequality $\varphi_{f}$ belongs to $l^{2}(G)$ (see [M] for a detailed description of this average operator). Necessarily $\varphi_{f}$ is given by

$$
\varphi_{f}=\left(\delta_{e}+\sum_{k=1}^{\infty} \frac{(-1)^{k} \chi_{k}}{n^{k}}\right) f(e)
$$

where $\chi_{k}$ denotes the characteristic function of $E_{k}$. The above function $\varphi_{f}$ cannot be in $l^{2}$ unless $q<n$.

On the other hand, if $q<n$, then the function $\varphi_{-\frac{1}{n}}$ defined in 2.15 by letting $f(e)=\left(1-\frac{q+1}{n+1}\right)$ is an idempotent in $C_{\text {red }}^{*}(G)$ and $\mathcal{N}_{2}$ is nothing but the corresponding subspace. (See $\left[\mathrm{T}_{2}\right]$ to see that $\varphi_{-\frac{1}{2}}$ is an idempotent of $C_{\text {red }}^{*}(G)$ and Section 4 for a more detailed description of $\mathcal{N}_{2}$.) Using [D-S](X 3.3) we may conclude that the spectral measure of $\left\{-\frac{1}{n}\right\}$ is nonzero for $q<n$, hence $g_{r}$ has a pole at $\gamma=-\frac{1}{n}$.

Finally suppose that $q=n$. We shall see in Section. 3 that $-\frac{1}{n}$ is a branch point for the analytic function $g_{r}(e)$ and

$$
\lim _{h \rightarrow 0} h g_{-\frac{1}{2}+h}(e)=0 .
$$

Since $g_{r}(e)$ has a Puisseux expansion in fractional powers powers of $\left(\gamma+\frac{1}{n}\right)$ in 
a neighbourhood of $-\frac{1}{n}, 2.16$ also implies that

$$
\lim _{\varepsilon-0} i \varepsilon g_{-\frac{1}{n}+i \varepsilon}(e)=0
$$

that is, $d m(\sigma)\left\{-\frac{1}{n}\right\}=0$ and $\mu$ has no(nontrivial) eigenspaces.

\section{$\S 3$. Description of $\operatorname{sp}(\mu)$}

We shall now investigate $\operatorname{sp}(\mu)$ in detail. It is clear from the above arguments that the point spectrum depends only on $q$ and $n$ and not the $p_{j}$. On the other hand, the continuous spectrum may or may not break up into many pieces depending on the $p_{j}$. We shall first state some results which are true in general, no

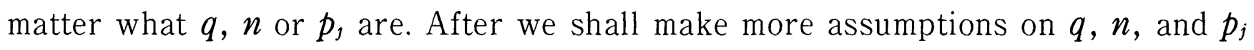
get the exact number of pieces of the continuous part of $\operatorname{sp}(\mu)$.

Let us define $I_{q+2}=\left(-\infty, p_{q+1}\left(\frac{n-1}{2 n}\right)\right], I_{k}=\left[p_{k}\left(\frac{n-1}{2 n}\right), p_{k-1}\left(\frac{n-1}{2 n}\right)\right]$ for $k=2, \ldots, q+1$ and $I_{1}=\left[p_{1}\left(\frac{n-1}{2 n}\right),+\infty\right)$. Since $\gamma^{\prime}(w)=$ $\sum_{1}^{q+1} \frac{ \pm z_{j}}{\sqrt{z_{j}^{2}+4 \frac{p_{j}^{2}}{n}}}-(q-1)$ we can see that, if at least two terms in the above summation are negative, $\gamma^{\prime}(w)$ will be negative. So that we have to consider first:

$$
\begin{array}{rlrl}
\gamma_{1} & =2 w+\frac{1}{2} \sum_{1}^{q+1}\left(\sqrt{z_{j}^{2}+4 \frac{p_{j}^{2}}{n}}-z_{j}\right) & \text { when } w \in I_{1} \\
\gamma_{q+2}=2 w-\frac{1}{2} \sum_{1}^{q+1}\left(\sqrt{z_{j}^{2}+4 \frac{p_{j}^{2}}{n}}+z_{j}\right) & \text { when } w \in I_{q+2} \\
\gamma_{k}=2 w-\frac{1}{2} \sum_{1}^{k-1}\left(\sqrt{z_{j}^{2}+4 \frac{p_{j}^{2}}{n}}+z_{j}\right)+\frac{1}{2} \sum_{k}^{q+1}\left(\sqrt{z_{j}^{2}+4 \frac{p_{j}^{2}}{n}}-z_{j}\right) & \text { when } w \in I_{k}
\end{array}
$$

It is clear that, when $w \in I_{k}(k=1, q+2)$, the corresponding $\gamma_{k}$ is the best possible choice in order to have $\gamma^{\prime}(w)$ positive. So that, whenever $\gamma_{k}^{\prime}$ is negative in $I_{k}$, no other curve can give a resolvent set for $w$ in $I_{k}$. On the other hand, when $\gamma_{k}^{\prime}$ is positive, other curves have to be considered in the corresponding $I_{k}$ namely we also check:

$$
\begin{array}{r}
\gamma_{1, h}=2 w+\frac{1}{2} \sum_{\substack{j \neq h \\
j=1}}^{q+1}\left(\sqrt{z_{j}^{2}+4 \frac{p_{j}^{2}}{n}}-z_{j}\right)-\frac{1}{2}\left(\sqrt{z_{h}^{2}+4 \frac{p_{h}^{2}}{n}}+z_{h}\right) \\
\quad \text { for } w \in I_{1}
\end{array}
$$




$$
\begin{gathered}
\gamma_{k}^{(h)=} 2 w-\frac{1}{2} \sum_{\substack{j=1 \\
j \neq h}}^{k-1}\left(\sqrt{z_{j}^{2}+4 \frac{p_{j}^{2}}{n}}+z_{\jmath}\right)+\frac{1}{2} \sum_{j=k}^{q+1}\left(\sqrt{z_{j}^{2}+4 \frac{p_{j}^{2}}{n}}-z_{j}\right)+ \\
+\frac{1}{2}\left(\sqrt{z_{h}^{2}+4 \frac{p_{h}^{2}}{n}}-z_{h}\right) \quad \text { for } w \in I_{k} \text { and } h \leq k-1 \\
\gamma_{k,(l)}=2 w-\frac{1}{2} \sum_{j=1}^{k-1}\left(\sqrt{z_{j}^{2}+4 \frac{p_{j}^{2}}{n}}+z_{j}\right)+\frac{1}{2} \sum_{\substack{j=k \\
j \neq l}}^{q+1}\left(\sqrt{z_{j}^{2}+4 \frac{p_{j}^{2}}{n}}-z_{j}\right)+ \\
\quad-\frac{1}{2}\left(\sqrt{z_{l}^{2}+4 \frac{p_{l}^{2}}{n}}+z_{l}\right) \quad \text { for } w \in I_{k} \text { and } l \geq k
\end{gathered}
$$

and finally

$$
\gamma_{q+2, h}=2 w-\frac{1}{2} \sum_{\substack{j \neq h \\ j=1}}^{q+1}\left(\sqrt{z_{j}^{2}+4 \frac{p_{j}^{2}}{n}}+z_{j}\right)+\frac{1}{2}\left(\sqrt{z_{h}^{2}+4 \frac{p_{h}^{2}}{n}}-z_{h}\right) \quad \text { for } w \in I_{q+2} .
$$

Let $\operatorname{sp}_{c}(\mu)$ denote the continuous part $\mathrm{sp}(\mu)$. We have the following

THEOREM 3.1. $\gamma_{1}$ and $\gamma_{q+2}$ are respectively convex and concave functions of $w . \gamma_{1}$ has a positive minimum, say $\rho_{1}$, and $\gamma_{q+2}$ has a maximum, say $\rho_{q+2}$, which is certainly negative when $q \geq n . \operatorname{sp}_{c}(\mu)$ is always contained in the interval $\left[\rho_{q+2}, \rho_{1}\right]$. For $w \in$ $I_{1}$ the only curve which can give a resolvent set is $\gamma_{1,1}$. When $p_{j}^{2}>\sum_{j=2}^{q+1} p_{j}^{2} \operatorname{sp}_{c}(\mu)$ consists of at least two parts. When $p_{j}^{2} \leq \sum_{j=2}^{q+1} p_{j}^{2}$ and $q \geq n$ no curve $\gamma_{q+2, k}$ can give a resolvent set in $I_{q+2}$.

Proof. The behaviour of $\gamma_{1}$ is very easy to check: $\gamma_{1}^{\prime \prime}$ is positive while $\lim _{w \rightarrow \pm \infty}$ $\gamma_{1}(w)=+\infty, \gamma_{1}^{\prime}(0)$ is negative and $\gamma_{1}(w)$ is positive for positive $w$. Also the behaviour of $\gamma_{q+2}$ is easy to check, being $\lim _{w \rightarrow \pm \infty} \gamma_{q+1}(w)=-\infty$ and $\gamma_{q+1}^{\prime \prime}(w)<0$, The last thing about $\gamma_{q+2}$ is the sign of its maximum $\rho_{q+2}$. Since $\gamma_{q+2}(0)=-\frac{1}{n}$ and $\gamma_{q+2}^{\prime}(0)=2 \frac{n-q}{n+1}$ we can see that, for $q \geq n$, the maximum occurs for a negative value of $w$ and hence is negative.

When $q=n \gamma_{q+2}^{\prime}(0)=0$. This means that the curve $\gamma_{q+2}$ gives the resolvent set for $\gamma<\frac{1}{n}$ but not for $\gamma>-\frac{1}{n}$. In other words, $\gamma$ is not a good parameter for the Riemann surface associated to $g_{\gamma}(e)$ in a neighborhood of $\gamma=-\frac{1}{n}$, so that $-\frac{1}{n}$ is a branch point for $g_{\gamma}(e)$. For $q<n$ the sign of $\rho_{q+2}$ is not, a priori, clear. It is obvious that $\operatorname{res}(\mu) \supset\left(-\infty, \rho_{q+2}\right) \cup\left(p_{1}+\infty\right)$ when $q \geq n$ while $\operatorname{res}(\mu)$ 
$\supset\left(-\infty,-\frac{1}{n}\right) \cup\left(-\frac{1}{n}, \rho_{q+2}\right) \cup\left(\rho_{1}+\infty\right)$ when $q<n$. we shall now analyze the curves $\gamma_{1, h}(h \neq 1)$ for $w \in I_{1}$. It is now convenient to introduce the following notation. Let

$$
\xi_{j}^{+}=\frac{+\sqrt{z_{j}^{2}+4 \frac{p_{j}^{2}}{n}}-z_{j}}{2 p_{j}} \text { and } \xi_{j}^{-}=\frac{-\sqrt{z_{j}^{2}+4 \frac{p_{j}^{2}}{n}}-z_{j}}{2 p_{j}}
$$

So that

$$
\gamma_{1, h}=2 w+\sum_{\substack{j \neq h \\ j=1}}^{q+1} p_{j} \xi_{j}^{+}+p_{h} \xi_{h}^{-}
$$

Since

$$
\left|\xi_{1}^{+} \xi_{h}^{-}\right|=\frac{1}{n} \cdot \frac{p_{1}}{p_{h}} \cdot \frac{\sqrt{z_{h}^{2}+4 \frac{p_{h}^{2}}{n}}+z_{h}}{\sqrt{z_{1}^{2}+4 \frac{p_{1}^{2}}{n}}+z_{1}}
$$

and $z_{h}>z_{1}$ we have

$$
\left|\xi_{1}^{+} \xi_{h}^{-}\right|>\frac{1}{n} \cdot \frac{p_{1}}{p_{h}} \cdot \frac{\sqrt{z_{1}^{2}+4 \frac{p_{h}^{2}}{n}}+z_{1}}{\sqrt{z_{1}^{2}+4 \frac{p_{1}^{2}}{n}}+z_{1}} \geq \frac{1}{n} \cdot \frac{p_{1}}{p_{h}} \cdot \frac{p_{h}}{p_{1}}=\frac{1}{n} .
$$

Hence, for the values of $\gamma$ corresponding to $\gamma_{1, h}$, we have

$$
\left|g_{r}\left(a_{1}^{k_{1}} a_{h}^{k_{2}} a_{1}^{k_{3}} a_{h}^{k_{4}} \cdots a_{1}^{k_{2 s-1}} a_{h}^{k_{2} s}\right)\right|^{2} \geq \frac{1}{n^{2 s}} .
$$

If we consider now the subgroup $G_{1, h}$, generated by $a_{1}$ and $a_{h}$, it is obvious that

$$
\sum_{x \in G_{1, h}}\left|g_{r}(x)\right|^{2}=+\infty .
$$

Hence none of the curves $\gamma_{1, h}$ give a resolvent set.

Consider now $\gamma_{1,1}$. Observe that

$$
\gamma_{1,1}=\sum_{j=2}^{q+1} p_{j} \xi_{j}^{+}-p_{1} \xi_{1}^{+}+p_{1}\left(\frac{n-1}{n}\right) .
$$

So that, for $w \rightarrow+\infty, \gamma_{1,1}$ approaches $p_{1}\left(\frac{n-1}{n}\right)$. A quick calculation shows that, for large $w, \gamma_{1,1}$ is less than $p_{1}\left(\frac{n-1}{n}\right)$ if $p_{j}^{2}>\sum_{j=2}^{q+1} p_{j}^{2}$ while it is greater than $p_{1}\left(\frac{n-1}{n}\right)$ if $p_{j}^{2} \leq \sum_{j=2}^{q+1} p_{j}^{2}$. Since $\gamma_{1,1}^{\prime}(0)$ is negative, we may conclude that, for $p_{1}^{2}>\sum_{j=2}^{q+1} p_{j}^{2}, \gamma_{1,1}$ has a minimum, say $\rho_{1,1}$, which is less than $p_{1}\left(\frac{n-1}{n}\right)$. Since 
$\gamma_{1,1}(0)=p_{1}\left(\frac{n-1}{n}\right)+\left(1-2 p_{1}\right)$, it is clear that, if $p_{1} \geq \frac{1}{2}, \gamma_{1,1}$ has only one minimum.

When $p_{1}<\frac{1}{2}$ we need some more conditions on the $p$, to understand the behaviour of $\gamma_{1,1}$. Let us consider now the curves $\gamma_{q+2, k}$. Suppose first $k \neq 1$. Remember that $\gamma_{q+2, k}=2 w+\sum_{j=1, j \neq k}^{q+1} p_{j} \xi_{j}^{-}+p_{k} \xi_{k}^{+}$and consider $\frac{d}{d w}\left|\xi_{1}^{-} \xi_{k}^{+}\right|$. A quick calculation shows that $\left|\xi_{1}^{-} \xi_{k}^{+}\right|$is decreasing for $2 w<\frac{p_{1}+p_{k}}{2} \frac{(n+1)^{2}}{n(n-1)}$. In particular, for negative $w$, we have

$$
\left|\xi_{1}^{-} \xi_{k}^{+}\right| \geq\left|\xi_{1}^{-}(0) \xi_{k}^{+}(0)\right|=\frac{1}{n} .
$$

Again, by considering the subgroup generated by $a_{1}$ and $a_{k}$, we can see that $\gamma_{q+2, k}(w)$ cannot give a resolvent set for negative $w$. For positive of $w$ is no longer true that $\gamma_{q+2, k}$ does not give a resolvent set (see Theorem 3.3).

Let now $\gamma_{q+2,1}=2 w+\sum_{j=2}^{q+1} p_{j} \xi_{j}^{-}+p_{1} \xi_{1}^{+}=\sum_{j=2}^{q+1} p_{j} \xi_{j}^{-}-p_{1} \xi_{1}^{-}+p_{1}\left(\frac{n-1}{n}\right)$. We can see that $\gamma_{q+2,1}$ tends to $p_{1}\left(\frac{n-1}{n}\right)$ for $w \rightarrow-\infty$. A quick computation shows that $\gamma_{q+2,1}$ is greater than $p_{1}\left(\frac{n-1}{n}\right)$ when $p_{j}^{2}>\sum_{2}^{q+1} p_{j}^{2}$, while $\gamma_{q+2,1}$ is less than $p_{1}\left(\frac{n-1}{n}\right)$ when $p_{1}^{2} \leq \sum_{2}^{q+1} p_{j}^{2}$. Again, by considering $\frac{d}{d w}\left|\xi_{j}^{-} \xi_{1}^{+}\right|$we see that $\left|\xi_{j}^{-} \xi_{1}^{+}\right|$is an increasing function of $w$ for $2 w<\frac{p_{1}+p_{1}}{2} \frac{(n+1)^{2}}{n(n-1)}$.

Suppose now $p_{1}^{2} \leq \sum_{2}^{q+1} p_{j}^{2}$. Consider the subset $\tilde{A}$ of $G$ consisting of all words $\tilde{x}$ of the form

$$
\tilde{x}=a_{1}^{k_{1}} a_{j_{1}}^{h_{1}} a_{12}^{k} a_{j_{2}}^{h_{2}} \cdots a_{1}^{k s} a_{s s}^{h_{s}} \quad(|x|=2 s) .
$$

Since $\lim _{w \rightarrow-\infty}\left|\xi_{j}^{-} \xi_{1}^{+}\right|=\frac{1}{n} \frac{p_{1}}{p_{1}}$, for $2 w \in I_{q+2}$, we have

$$
\sum_{\tilde{x} \in \tilde{A}}\left|g_{\gamma}(\tilde{x})\right|^{2}=\sum_{s=0}^{\infty} n^{2 s}\left(\sum_{j=2}^{q+1}\left|\xi_{j}^{-} \xi_{1}^{+}\right|^{2}\right)^{s}=+\infty
$$

since the ratio of the geometric series on right hand side of 3.4 is greater than 1 . Let now consider any $w$ in $I_{q+2}$ and let $\gamma$ be the corresponding value of $\gamma_{q+2,1}(w)$. We have

$$
\left\|g_{\gamma}\right\|_{2}^{2} \geq \sum_{\tilde{x} \in \tilde{A}}\left|g_{\gamma}(\tilde{x})\right|^{2}=+\infty
$$

and $\gamma_{q+2,1}$ does not give a resolvent set.

On the other hand, when $p_{1}^{2}>\sum p_{j}^{2}, \gamma_{q+2,1}$ is increasing for $w$ sufficiently small. Suppose now that for some $w_{0} \in I_{q+2} \gamma_{q+2,1}^{\prime}\left(w_{0}\right)$ is negative and consider 
$w_{1}>w_{0}$. Let $\gamma_{0}=\gamma_{q+2,1}\left(w_{0}\right)$ and $\gamma_{1}=\gamma_{q+2,1}\left(w_{1}\right)$. Observe that $\left|\xi_{j}^{-}\right|$is increasing, as well as $\left|\xi_{j}^{-} \xi_{1}^{+}\right|$, and $g_{\gamma_{1}}(x)$ is nothing but the product of $\frac{1}{2 w}, \xi_{j}^{-}$and $\xi_{1}^{+}$. In computing the $l^{2}$ norm of $g_{\gamma_{1}}(x)$ we may of course restrict our attention to the words $x$ which do not end with a power of $a_{1}$, let us denote by $A_{1}$ this subset of $G$. We have

$$
\left\|g_{\gamma_{1}}\right\|_{2}^{2}=\left(\frac{1}{2 w_{1}}\right)^{2}\left\{1+\left(n\left|\xi_{1}\right|^{2}+1\right) \sum_{x \in A_{1}}\left|g_{\gamma_{1}}(x)\right|^{2}\right\}
$$

but the right hand side of 3.6 is infinite, since $g_{\gamma_{0}}$ does not belong to $l^{2}(G)$. This argument proves that, for $w$ in $I_{q+2}, \gamma_{q+2,1}$ can only behave in two ways: either it is everywhere increasing, or it increases up to a maximum, say $\rho_{q+2,1}$ which is greater than $p_{1}\left(\frac{n-1}{n}\right)$ and after decreases. To ensure that $\rho_{q+2,1}$ occurs, observe that $\gamma_{q+2,1}^{\prime}(w)$ is negative $w=p_{q+1}\left(\frac{n-1}{n}\right)$. We may conclude that, when

$$
p_{1}^{2}>\sum_{j=2}^{q+1} p_{j}^{2}, \quad \operatorname{sp}_{c}(\mu) \subset\left[\rho_{q+2} \rho_{1,1}\right] \cup\left[\rho_{q+2,1}, \rho_{1}\right]
$$

In the above theorem we didn't investigate the curves $\gamma_{k}$ (and $\gamma_{k}^{(h)} \gamma_{k,(h)}$ ) in $I_{k}$. Their behaviour depends of course on the $p_{j}$, but the general law seems to be very complicated. We shall now exibit three "extreme" cases: in the first one they are all decreasing while in the two others every $\gamma_{k}$ gives a resolvent set.

THEOREM 3.2. Suppose that $p_{1} \leq 2 p_{q+1}, q \geq n$, and, if $p_{1} \neq p_{2}$, suppose that at least one of the following conditions hold;

i) $\quad \sum_{n=2}^{q+1} p_{j}^{2}>n p_{1}^{2}$

or

ii) $\left(\frac{p_{1}+p_{2}}{2}\right)\left(\frac{n+1}{n-1}\right)^{2} \leq p_{1}$ and $n \geq 4$.

Then $\operatorname{sp}(\mu)=\left[p_{q+2}, \rho_{1}\right]$.

Proof. We must check the curves $\gamma_{k}$ in $I_{k}$ first.

We have

$$
\gamma_{k}^{\prime}=-(q-1)+\sum_{j=k}^{q+1} \frac{z_{j}}{\sqrt{z_{j}^{2}+4 \frac{p_{j}^{2}}{n}}}+\sum_{j=1}^{k-1} \frac{-z_{j}}{\sqrt{z_{j}^{2}+4 \frac{p_{j}^{2}}{n}}}<
$$




$$
\begin{gathered}
<-(q-1)+\sum_{j=k}^{q+1} \frac{\left(p_{k-1}-p_{j}\right)\left(\frac{n-1}{n}\right)}{\sqrt{\left(p_{k-1}-p_{j}\right)^{2}\left(\frac{n-1}{n}\right)^{2}+4 \frac{p_{j}^{2}}{n}}}+ \\
+\sum_{j=1}^{k+1} \frac{\left(p_{j}-p_{k}\right)\left(\frac{n-1}{n}\right)}{\sqrt{\left(p_{j}-p_{k}\right)^{2}\left(\frac{n-1}{n}\right)^{2}+4 \frac{p_{j}^{2}}{n}}} .
\end{gathered}
$$

Since $p_{k-1}-p_{j} \leq p_{1}-p_{j} \leq p_{j}$ for $j \geq k$ while $p_{j}-p_{k} \leq p_{j}$ for $j<k$ we get

$$
\gamma_{k}^{\prime}<-(q-1)+(q+1)\left(\frac{n-1}{n+1}\right)=2 \frac{(n-q)}{n+1} \leq 0 \quad \text { since } q \geq n \text {. }
$$

Of course none of the curves $\gamma_{k}^{(h)}$ or $\gamma_{k,(h)}$ can give a resolvent set, since $\gamma_{k}^{\prime}<0$ everywhere. The last curve we must check is $\gamma_{1,1}$, It is obvious that this problem disappears when $p_{1}=p_{2}$, of course we shall choose $\xi_{1}=\xi_{2}=\xi_{1}^{+}$. Recall that

$$
r_{1,1}=2 w+\sum_{2}^{q+1} p_{j} \xi_{1}^{+}+p_{1} \xi_{1}^{-}
$$

Suppose now that i) holds and consider $\left|\xi_{1}^{-} \xi_{1}^{+}\right|$.

We have:

$$
\left|\xi_{1}^{-} \xi_{j}^{+}\right|=\frac{1}{4 p_{1} p_{j}}\left(\sqrt{z_{1}^{2}+4 \frac{p_{1}^{2}}{n}}+z_{1}\right) \cdot\left(\sqrt{z_{j}^{2}+4 \frac{p_{j}^{2}}{n}}-z_{j}\right)
$$

and, since $w$ is greater than $p_{1}\left(\frac{n-1}{2 n}\right)$.

$$
\begin{aligned}
\left|\xi_{i}^{-} \xi_{j}^{+}\right|>\frac{p_{j}}{n p_{1}} \frac{\sqrt{z_{j}^{2}+4 \frac{p_{j}^{2}}{n}}-z_{j}}{\sqrt{z_{1}^{2}+4 \frac{p_{j}^{2}}{n}-z_{1}}} \geq \\
\\
\quad \geq \frac{p_{1}}{n p_{1}} \frac{\sqrt{\left(p_{1}-p_{j}\right)^{2}\left(\frac{n-1}{n}\right)^{2}+4 \frac{p_{j}^{2}}{n}}-\left(p_{1}-p_{j}\right)^{2}\left(\frac{n-1}{n}\right)}{\frac{2 p_{j}}{\sqrt{n}}}
\end{aligned}
$$

Again, since $p_{1}-p_{j} \leq p_{j}$, we have

$$
\left|\xi_{j}^{+} \xi_{1}^{-}\right|>\frac{p_{j}}{p_{1}} n^{-\frac{3}{2}}
$$

and hence

$$
\sum_{s=0}^{\infty}\left(\sum_{j=2}^{q+1} n^{2}\left|\xi_{j}^{+} \xi_{1}^{-}\right|^{2}\right)=+\infty
$$

which implies that $\gamma_{1,1}$ cannot give a resolvent set. 
Of course condition i) is quite unsatisfactory for large values of $n$. Suppose now that $n$ is large enough (at least $n \geq 4$ ) to ensure that condition ii) holds.

One can see that $\frac{d}{d w}\left|\xi_{1}^{-} \xi_{j}^{+}\right|$is positive whenever $2 w>\left(\frac{p_{1}+p_{j}}{2}\right) \frac{(n+1)^{2}}{n(n-1)}$. Condition ii) ensures that, for every $j$ and for every $w$ in $I_{1}\left|\xi_{1}^{-} \xi_{j}^{+}\right|$is increasing.

Suppose now that $\gamma_{1,1}^{\prime}\left(w_{2}\right)>0$ for some $w_{2}>p_{1}\left(\frac{n-1}{2 n}\right)$. At $w=w_{2}$ we must have

$$
\sum_{s=0}^{+\infty}\left(\sum_{j=2}^{q+1} n^{2}\left|\xi_{1}^{-}\left(w_{2}\right) \xi_{j}^{+}\left(w_{2}\right)\right|^{2}\right)^{s}<+\infty
$$

and hence

$$
n^{2} \sum_{j=2}^{q+1}\left|\xi_{1}^{-}\left(w_{2}\right) \xi_{j}^{+}\left(w_{2}\right)\right|^{2}<1
$$

but 3.9 implies that, for $w_{1}=p_{1}\left(\frac{n-1}{2 n}\right)$

$$
n^{2} \sum_{j=2}^{q+1} \frac{1}{n}\left|\xi_{j}^{+}\left(w_{1}\right)\right|^{2}<1 .
$$

Again, since $\left(p_{1}-p_{j}\right) \leq p_{j}$. we have $\left|\xi_{j}^{+}\left(w_{1}\right)\right| \geq \frac{1}{n}$, hence $\frac{q}{n} \leq \sum_{j=2}^{q+1} n\left|\xi_{j}^{+}\left(w_{1}\right)\right|^{2}$ $<1$ which is a contradiction if $q \geq n$.

Remark. In general we cannot ensure that $\gamma_{1,1}$ does not give a resolvent set, even if $p_{1}^{2} \leq \sum_{j=2}^{q+1} p_{j}^{2}$. Consider in fact $G=\boldsymbol{Z}_{3} * \boldsymbol{Z}_{3} * \boldsymbol{Z}_{3}$. Set $p_{3}=p_{2}=\frac{1}{\sqrt{2}+2}$ and $p_{1}=\frac{1}{1+\sqrt{2}}$. Then $\gamma_{1,1}^{\prime}\left(\frac{9}{4}\left(\frac{\sqrt{2}}{4}\right)\right)$ is positive even if $\gamma_{1,1}^{\prime}<0$ for $w<$ $\frac{1}{4(1+\sqrt{2})}=p_{1}\left(\frac{n-1}{2 n}\right)$ and also $\gamma_{1,1}^{\prime}<0$ for $w \rightarrow+\infty$.

THEOREM 3.2. Fix $q$ and choose $p_{1}, \ldots, p_{q+1}$ so that

$$
p_{j} \geq(j+1) p_{j+1} \quad \text { for } j=1, \ldots, q .
$$

Suppose now that $n$ is bigger than $q$, and is also large enough to ensure that

$$
\frac{p_{1}}{p_{q+1}} \frac{2 \sqrt{n}}{(n-1)}<1
$$


Then $\operatorname{sp}_{c}(\mu)$ consists of exactly $q+1$ pieces.

Proof. Observe first that, since $q<n,-\frac{1}{n}$ belongs to $\operatorname{sp}(\mu)$ and corresponds to an eigenspace of $\mu$, or to a subrepresentation, say $\pi_{-\frac{1}{n}}$, of the left regular representation of $G$. We shall see in Section 4 that the orthogonal projection onto this subspace is given by right convolution with a function $\varphi$, whose value at the identity is $\frac{n-q}{n+1}$. We can think of $\varphi$ as representing an element of the $K$-theory of $C_{\mathrm{reg}}^{*}(G)$, and we can also see that the continuous dimension of $\pi_{-\frac{1}{n}}$ is $\frac{n-q}{n+1}$ (see $[\mathrm{KS}]$ for the definition of the continuous dimension and [L] for more informations on the $K$-theory of $C_{\text {reg }}^{*}(G)$ ). We also know from [L] that any other subrepresentation of the regular representation which corresponds to a projection in $C_{\text {reg }}^{*}(G)$ has continuous dimension equal to an integral multiple of $\frac{1}{n+1}$.

Suppose we know that $\operatorname{sp}_{c}(\mu)$ breaks up into at least $q+1$ pieces. In correspondence to any of these pieces we have a subrepresentation of the regular which also corresponds to a projection in $C_{\mathrm{reg}}^{*}(G)$. The continuous dimension of $\pi_{-\frac{1}{n}}$ is $\frac{n-q}{n+1}$ and the regular representation itself has continuous dimension 1 so we may conclude that $\operatorname{sp}_{c}(\mu)$ consists of exactly $q+1$ pieces.

Hence all we have to prove is that $\operatorname{sp}_{c}(\mu)$ consists of at least $q+1$ pieces. Define, for $2 \leq k \leq q+1$.

$$
\begin{aligned}
A_{k}=-(q-1) & +\sum_{j=1}^{k-1} \frac{\left(p_{j}-p_{k}\right)\left(\frac{n-1}{n}\right)}{\sqrt{\left(p_{j}-p_{k}\right)^{2}\left(\frac{n-1}{n}\right)^{2}+4 \frac{p_{j}^{2}}{n}}}+ \\
& +\sum_{j=k+1}^{q+1} \frac{\left(p_{k}-p_{j}\right)\left(\frac{n-1}{n}\right)}{\sqrt{\left(p_{k}-p_{j}\right)^{2}\left(\frac{n-1}{n}\right)^{2}+4 \frac{p_{j}^{2}}{n}}} \\
= & \gamma_{k}^{\prime}\left(p_{k}\left(\frac{n-1}{2 n}\right)\right)=\gamma_{k+1}^{\prime}\left(p_{k}\left(\frac{n-1}{2 n}\right)\right)
\end{aligned}
$$

and check the sign of $A_{k}$. Since $p_{j} \geq(j+1) p_{j+1}$ and $p_{k}-p_{j} \geq(j-1) p_{j}$ for $j \geq k+1$, one has: 


$$
\begin{aligned}
& A_{k} \geq-(q-1)+\sum_{j=1}^{k-1} \frac{\left(p_{j}-p_{j+1}\right)\left(\frac{n-1}{n}\right)}{\sqrt{\left(p_{j}-p_{j+1}\right)^{2}\left(\frac{n-1}{n}\right)^{2}+4 \frac{p_{j}^{2}}{n}}}+ \\
&+\sum_{k+1}^{q+1} \frac{(j-1) p_{j}\left(\frac{n-1}{n}\right)}{\sqrt{(j-1)^{2} p_{j}^{2}\left(\frac{n-1}{n}\right)^{2}+4 \frac{p_{j}^{2}}{n}}} \\
& \geq-(q-1)+\sum_{j=1}^{k+1} \frac{j p_{j+1}\left(\frac{n-1}{n}\right)}{\sqrt{j^{2} p_{j+1}^{2}\left(\frac{n-1}{n}\right)^{2}+4 \frac{p_{j}^{2}}{n}}}+\sum_{j=k}^{q} \frac{j}{\sqrt{j^{2}+\frac{j n}{(n-1)^{2}}}} \\
&>-(q-1)+\sum_{j=1}^{k+1} \frac{j}{\sqrt{j^{2}+\frac{j n}{(n-1)^{2}}\left(\frac{p_{1}}{p_{q+1}}\right)^{2}}}+\sum_{j=k}^{q} \frac{j}{\sqrt{j^{2}+\frac{4 n}{(n-1)^{2}}}} \\
&>-(q-1)+\int_{0}^{q} \frac{x d x}{\sqrt{x^{2}+\frac{4 n}{(n-1)^{2}}\left(\frac{p_{1}}{p_{q+1}}\right)^{2}}}
\end{aligned}
$$

hence

$$
A_{k}>-(q-1)+\sqrt{q^{2}+\frac{4 n}{(n-1)^{2}}\left(\frac{p_{1}}{p_{q+1}}\right)^{2}}-2\left(\frac{p_{1}}{p_{q+1}}\right) \frac{\sqrt{n}}{(n-1)}
$$

and, under our hypothesis, the above quantity is positive.

Observe that, for each $k, 2 \leq k \leq q+1, \gamma_{k}$ gives rise to a piece of the resolvent set which is contained in $\left[p_{q+2}, p_{1}\right]$. Since two different $\gamma_{k}$ cannot give rise to overlapping pieces of the resolvent set, we may conclude that $\operatorname{sp}_{c}(\mu)$ breaks up into at least $q+1$ (hence exactly $q+1$ ) pieces.

The next question is: suppose that $q \geq n$. Since no information on the dimension of $\pi_{-\frac{1}{n}}$ is available, what is the largest possible number for the connected components of $\operatorname{sp}(\mu)$ ? First of all, according with the $K$-theory of $G$. we cannot get more than $(n+1)$ components. If we look at the quantities $A_{k}$, it is not hard to show that $A_{k}$ is negative until $k$ is bigger or equal to $1+\left[\frac{n+1}{2}\right]$. Nevertheless it is still possible to obtain exactly $(n+1)$ pieces for $\operatorname{sp}(\mu)$ by letting the $p_{j}$ be far enough from each other.

We thank Tim Steger for suggesting us the following 
THEOREM 3.3. Suppose that $q \geq n$. Then there exists a choice of the $p_{j}$ 's in such a way that $\operatorname{sp}(\mu)=\operatorname{sp}_{c}(\mu)$ consists of exactly $n+1$ intervals.

Proof. Suppose that $p_{q+1} \leq p_{q} \leq \cdots \leq p_{n+1}$ are given, we shall construct $p_{n}, \ldots, p_{1}$. In this construction we shall drop the hypothesis $\sum_{1}^{q+1} p_{1}=1$ which is really not needed in order to check the sign of the derivative of $\gamma_{k}$. At the end we shall get curves $\gamma_{k} k=n+1, \ldots, 2$, which have positive derivative at some point of $I_{k}=\left[p_{k}\left(\frac{n-1}{2 n}\right), p_{k-1}\left(\frac{n-1}{2 n}\right)\right]$ and we shall renormalize the $p_{j}$ to get the right probabilities.

Let us denote by $\phi_{j}$ the derivative of $p_{j} \xi_{j}^{+}$namely

$$
\phi_{j}=\frac{z_{j}}{\sqrt{z_{j}^{2}+4 \frac{p_{j}^{2}}{n}}} \quad j=1, \ldots, q+1
$$

so that

$$
\gamma_{k}^{\prime}=-(q-1)+\sum_{j=k}^{q+1} \phi_{j}+\sum_{j=1}^{k-1}-\phi_{j}
$$

Let us construct $p_{n}$ from $p_{n+1}$. Choose $\varepsilon$ in such a way that

$$
2\left(1-\frac{n}{n+1}\right)-(q+1) \varepsilon>0 .
$$

Since $\lim _{w \rightarrow+\infty} \phi_{n+1}=1$ there exists $f>\frac{n-1}{2 n}$ such that

$$
\phi_{n+1}\left(p_{n+1} f\right)>1-\varepsilon .
$$

Also, for $k>n+1$ and $w$ in $I_{n+1}$, we have $\phi_{k}(w)>\phi_{n+1}(w)$ hence

$$
\phi_{k}\left(p_{n+1} f\right)>1-\varepsilon \text { for } k \geq n+1 .
$$

On the other hand one has

$$
\lim _{p_{n \rightarrow+\infty}}\left(-\phi_{n}\right)\left(p_{n+1} f\right)=\lim _{p_{n \rightarrow+\infty}} \frac{p_{n}\left(\frac{n-1}{n}\right)-2 p_{n+1} f}{\sqrt{\left(p_{n}\left(\frac{n-1}{n}\right)-2 p_{n+1} f\right)^{2}+\frac{4 p_{n}^{2}}{n}}}=\frac{n-1}{n+1}
$$

so that it is also possible to choose $p_{n}$ in such a way that

$$
-\phi_{n}\left(p_{n+1} f\right)>\frac{n-1}{n+1}-\varepsilon
$$

Again, since $-\phi_{j}(w) \geq-\phi_{n}(w)$ for $j<n$ and $w \geq p_{n+1}\left(\frac{n-1}{2 n}\right)$, we get 


$$
-\phi_{,}\left(p_{n+1} f\right)>\frac{n-1}{n+1}-\varepsilon \text { for every } j \leq n .
$$

So that, starting from $p_{n+1}$, it is possible to choose $p_{n}$ large enough in such a way that $p_{n+1} f<p_{n}$ and 3.15 holds. Hence, for $w=p_{n+1} f \in I_{n+1}$ one has:

$$
\begin{gathered}
\gamma_{n+1}^{\prime}\left(p_{n+1} f\right)=-(q-1)+\sum_{j=n+1}^{q+1} \phi_{j}\left(p_{n+1} f\right)+\sum_{j=1}^{n}-\phi_{j}\left(p_{n+1} f\right) \geq \\
\geq 2\left(1-\frac{n}{n+1}\right)-\varepsilon(q+1)>0
\end{gathered}
$$

Suppose now that $p_{q+1}, \ldots, p_{j}$ are given $(j \geq n+1)$ and let us find $p_{j-1}$. Choose $\varepsilon$ in such a way that

$$
2\left(1-\frac{j-1}{n+1}\right)-\varepsilon(q+1)>0
$$

(observe that 3.16 cannot be satisfied when $j \geq n+2$ !). Since $\lim _{w \rightarrow+\infty} \phi_{j}=1$ we can find $f_{j}$ such that $f_{j} p_{j}>p_{j}$ and $\phi_{j}\left(p_{j} f_{j}\right)>1-\varepsilon$. Also, since $\lim _{p j-1 \rightarrow-\infty}-\phi_{j-1}\left(p_{j} f_{j}\right)=\frac{n-1}{n+1}, p_{j-1}$ can be chosen in such a way that $p_{j-1}>p_{j} f_{j}$ and $-\phi_{j-1}\left(p_{j} f_{j}\right) \geq \frac{n-1}{n+1}-\varepsilon$. Again, for $w=p_{j} f_{j}$ we have

$$
\gamma_{j}^{\prime}\left(p_{j} f_{j}\right) \geq 2-\frac{2(j-1)}{n+1}-\varepsilon(q+1)>0 \text {. }
$$

If we consider now the probabilities $p_{j}$ which are obtained by normalizing the $p_{j}$, we can see that each of the corresponding $\tilde{\gamma}_{k}(k=n+1, \ldots, 2)$ has positive derivative in a suitable point of $\tilde{I}_{k}$. Arguing as in the proof of Theorem 3.2 we may conclude that $\operatorname{sp}(\mu)$ consists of exactly $n+1$ intervals.

\section{The irreducible representations}

This section is devoted to the description of the measure $d m(\sigma)$, of the positive definite functions $\left\{\varphi_{\sigma}(x)\right\}_{\sigma}$ and of the unitary irreducible representations. The construction is essentially the one given in [S]. We shall not give the proof of the statements which can be deduced arguing exactly as in [S].

In order to produce $\left\{\varphi_{\sigma}(x)\right\}_{\sigma \in \mathrm{sp}(\mu)}$ we need to know the following

Lemma 4.1. Let $q>n$ and $\sigma \in \operatorname{sp}(\mu)$. Then $g_{\sigma \pm i 0}(x) \neq \infty$ and $g_{\sigma \pm t 0}$ is a continuous function of $\sigma$. Moreover, if $g_{\sigma+t 0}(e)=g_{\sigma-10}(e)$ then $\sigma$ is a branch point for the analytic function $g_{\gamma}(e)$. 
Proof. As noted in Section 2 (2.14) $w(\gamma)$ cannot be zero unless $q \leq n$. So if $g_{\sigma \pm 20}(x)=\infty$ for some $x$, we must have $\xi_{j}(\sigma \pm i 0)=\infty$ for same $j$. The same argument used in Section 2 (2.8) shows that $g_{\sigma \pm i 0}(x)$ has the form given in 2.10 . In particular, $g_{\sigma \pm 10}(x)$ is finite. To see that $g_{\sigma \pm 10}(x)$ is continuous, fix any point $\sigma_{0}$ $\in \operatorname{sp}(\mu)$. Since $g_{r}(x)$ is analytic in the upper half-plane and $g_{\sigma+i 0}(x) \neq \infty$, we are sure that, at $\gamma=\sigma_{0}, g_{\gamma}$ has a Pouisseux expansion without negative powers, hence $g_{\sigma+\imath 0}$ is a continuous function of $\sigma$ in a neighborhood of $\sigma_{0}$. Keeping these facts in mind, the arguments used in [S] apply to show the last statement.

Let us consider now the case $q \leq n$. In Section 2 we saw that the only possible value of $\sigma$ for which $g_{\sigma \pm 10}(x)=\infty$ is $-\frac{1}{n}$. On the other hand, when $g_{\sigma \pm i 0}(x)$ is finite, one can argue as in Lemma 4.1 to get similar results for $g_{\sigma \pm i 0}(x)$. Hence we have a sort of complementary result for $q \leq n$.

Lemma 2. Let $q<n$. Then, if $\sigma \neq-\frac{1}{n}$, all the statements of Lemma 1 are true. If $\sigma=-\frac{1}{n}$ then $\operatorname{dm}(\sigma)=\frac{n-q}{n+1}$ and the corresponding positive definite function is the function $\varphi_{-\frac{1}{n}}$ given in 2.15 with $\varphi_{-\frac{1}{n}}(e)=\frac{n-q}{n+1}$.

When $q=n$ the point $\sigma=-\frac{1}{n}$ is a branch point for $g_{\gamma}(e)$ and $g_{-\frac{1}{n} \pm i 0}(e)$ is infinite. For all the others values of $\sigma g_{\sigma \pm i 0}(x)$ is a continuous function of $\sigma$.

Proof. Suppose that $q<n$. As we said before, we may restrict our attention to $\sigma=-\frac{1}{n}$. As noted in Theorem 3.1 the function $\gamma_{q+2}(w)$ gives the (real) resolvent set in a (real) neighborhood of $w=0$. Hence, in a (real) neighborhood $\gamma=-\frac{1}{n}$, we have $g_{\gamma}(e)=\frac{1}{2 w_{q+2}(\gamma)}$. We may now compute $d m\left(-\frac{1}{n}\right)$. In fact one has:

$$
d m\left(-\frac{1}{n}\right)=\lim _{\varepsilon \rightarrow 0^{+}}\left(-\frac{1}{n}+i \varepsilon\right) g_{-\frac{1}{n}+i \varepsilon}(e)
$$

But the above limit can be computed on the real axis, so that we get

$$
d m\left(-\frac{1}{n}\right)=\left.\frac{1}{2}\left(\frac{1}{\frac{d w_{g+2}}{d \gamma}}\right)\right|_{\gamma=-\frac{1}{n}}=\frac{1}{2}\left(\frac{d \gamma_{q+2}}{d w}\right)_{w=0}=\frac{n-q}{n+1}
$$

As we said in Section 2. when $q<n, \mu$ has an eigenspace $B$ which contains the subspace $\mathcal{N}_{2}$ of $l^{2}$ functions having zero average over each polygon. The projection 
onto $\mathcal{N}_{2}$ is given by right convolution with the function

$$
\varphi-\frac{1}{n}=\left(1-\frac{q+1}{n+1}\right)\left(\delta_{e}-\sum_{m=1}^{\infty}\left(-\frac{1}{n}\right)^{m} \chi_{m}\right) .
$$

Let us now consider $B$. The functional calculus says that the orthogonal projection $F$ onto $B$ is given by

$$
F=\frac{1}{2 \pi i} \int_{C} \frac{1}{\lambda+\frac{1}{n}} E(d \lambda)
$$

where $E$ is the resolution of the identity for $M$ and $C$ is a sufficiently small circle about $-\frac{1}{n}$. Since $F$ belongs to $C_{\mathrm{reg}}^{*}(G)$ we have $F(f)=h * f$ for a suitable positive definite $f$. On the other hand, one has

$$
\left\langle F\left(\delta_{e}\right), \delta_{e}\right\rangle=f(e)=d m\left(-\frac{1}{n}\right)=\frac{n-q}{n+1}=\varphi-\frac{1}{n}(e) .
$$

Hence $f \equiv \varphi\left(\right.$ and $\left.B=\mathcal{N}_{2}\right)$.

Let now $q=n$. Again, by considering $\gamma_{q+2}$ as in Theorem 3.1, we see that $\gamma=-\frac{1}{n}$ is a branch point for $g_{r}(e)$. We remark that, since $\left.\frac{d \gamma_{q+2}}{d w}\right|_{w=0}=0$, $d m\left(-\frac{1}{n}\right)$ is zero.

Let $S$ denote the set of branch points of $g_{r}(e)$. Since $g_{\gamma}(e)$ is an algebraic function, $S$ is finite. For any $\sigma \in \operatorname{sp}_{c}(\mu) \backslash S$ define

$$
\varphi_{\sigma}(x)=\frac{g_{\sigma+i 0}(x)-g_{\sigma-i 0}(x)}{g_{\sigma+i 0}(e)-g_{\sigma-i 0}(e)}
$$

and

$$
d m(\sigma)=-\frac{1}{\pi}\left(g_{\sigma+i 0}(e)-g_{\sigma-i 0}(e)\right) d \sigma .
$$

Then the functional calculus says that

$$
\begin{aligned}
& \delta_{e}(x)=\int_{s p(\mu)} \varphi_{\sigma}(x) d m(\sigma) \text { when } q \geq n \\
& \delta_{e}(x)=\varphi_{-\frac{1}{n}}(x)+\int_{s p_{c}(\mu)} \varphi_{\sigma}(x) d m(\sigma) \text { when } q<n .
\end{aligned}
$$

In fact, all the functions $\varphi_{\sigma}$ involved, are two sided eigenfunctions of $\mu$ (with eigenvalue $\sigma$ ) and the sum in 4.4 is an orthogonal sum. Using the functional calculus one can argue as in $[\mathrm{S}]$ to see that $-\frac{1}{\pi}\left\{g_{\sigma+i 0}(x)-g_{\sigma-\imath 0}(x)\right\}$ is positive 
definite for $\sigma \in \operatorname{sp}_{c}(\mu)$, hence $\varphi_{\sigma}(x)$ is positive definite for $\sigma \in \operatorname{sp}_{c}(\mu) \backslash S$. Corresponding to any $\varphi_{\sigma}\left(\sigma \in \operatorname{sp}_{c}(\mu) \backslash S\right)$ we may associate a continuous unitary representation of $G$, say $\pi_{\sigma}$.

When $\sigma=\left\{-\frac{1}{n}\right\}$ then the corresponding representation in $l^{2}(G)$ and hence is reducible (see [FTC]).

When $\sigma \neq\left\{-\frac{1}{n}\right\}$ then the corresponding $\pi_{\sigma}$ is realized in a standard Hilbert space $\mathscr{H}_{\sigma}$, which can be thought to be the completion of the space of left translates of $\varphi_{\sigma}$. For any finitely supported functions $f$ and $g$ we have:

$$
\begin{gathered}
f \mapsto f_{\sigma}=f * \varphi_{\sigma}, \quad \pi_{\sigma}(x) f_{\sigma}=\left(\delta_{x} * f\right)_{\sigma} \\
\left(f_{\sigma}, g_{\sigma}\right)_{\sigma}=\left(f * \varphi_{\sigma}, g\right)
\end{gathered}
$$

(, ) denotes the inner production $l^{2}(G)$ and $(,)_{\sigma}$ the one in $H_{\sigma}$.

Also, because of 4.3 and 4.4 , we have

$$
(f, g)=\int_{s p(\mu)}\left(f * \varphi_{\sigma}, g\right) d m(\sigma)=\int_{s p_{c}(\mu)}\left(f_{\sigma}, g_{\sigma}\right)_{\sigma} d m(\sigma) \text { when } q \geq n
$$

and

$$
(f, g)=\left(f * \varphi_{-\frac{1}{n}}, g\right)+\int_{s p_{c}(\mu)}\left(f_{\sigma}, g_{\sigma}\right)_{\sigma} d m(\sigma) \text { when } q<n
$$

Let $\sigma \in \operatorname{sp}(\mu) \backslash\left\{-\frac{1}{n}\right\}$ and let $g_{\gamma}(x)$ be equal to $(\gamma-\mu)^{-1}(x)$ at $\gamma=\sigma+i \varepsilon$, so that $g_{\gamma}(e)=\frac{1}{2 w(\gamma)}$. In $[\mathrm{S}]$ it is proved that if $\lim _{\varepsilon \rightarrow 0+} w(\gamma) \neq \lim _{\varepsilon \rightarrow 0-} w(\gamma) \neq 0 \neq \infty$ then the corresponding representation $\pi_{0}$ is irreducible. The same arguments used in [S] also apply to our case. (Observe that when $\sigma=-\frac{1}{n}$ we have $\lim _{\varepsilon \rightarrow 0 \pm} w(\gamma)$ $=0)$. Namely, we have the following.

THEOREM 4.1. Suppose that $\sigma \in \operatorname{sp}(\mu) \backslash\left\{S \cup\left\{-\frac{1}{n}\right\}\right\}$. Then the corresponding representation $\pi_{0}$ on $H_{\sigma}$ is irreducible.

Sketch of the Proof.

1) Let $Q(\sigma)=\left\{\phi \in H_{\sigma}: \pi_{\sigma}(\mu) \phi=\sigma \phi\right\}$. Observe that $\varphi_{\sigma}$ belongs to $Q(\sigma)$ and recall that, if $Q(\sigma)$ is one dimensional, then $\pi_{0}$ is irreducible.

2) Let $Q_{\sigma}$ be the orthogonal projection onto $H_{\sigma}$, the functional calculus says 
that

$$
Q_{\sigma}=\lim _{\varepsilon \rightarrow 0+} i \varepsilon\left(\sigma+i \varepsilon-\pi_{\sigma}(\mu)\right)^{-1} .
$$

3) Observe that $Q_{\sigma}$ can be computed for large values of $\varepsilon$ and then take the analytic continuation. Let $\sigma^{\prime}=\sigma+i \varepsilon$ and $g_{\sigma^{\prime}}=(\sigma+i \varepsilon-\mu)^{-1}$. Then for large values of $\varepsilon$ we have

$$
\left[\sigma+i \varepsilon-\pi_{\sigma}(\mu)\right]^{-1}=\pi_{\sigma}\left\{(\sigma+i \varepsilon-\mu)^{-1}\right\}
$$

hence

$$
\begin{aligned}
\left(Q_{\sigma}\left(\delta_{x} * \varphi_{\sigma}\right), \delta_{y} * \varphi_{\sigma}\right)_{\sigma} & =\lim _{\varepsilon \rightarrow 0+} i \varepsilon\left(\pi_{\sigma}\left\{\left(\sigma^{\prime}-\mu\right)^{-1}\right\}\left[\delta_{x} * \varphi_{\sigma}\right], \delta_{y} * \varphi_{\sigma}\right)_{\sigma} \\
& =\lim _{\varepsilon \rightarrow 0+} i \varepsilon\left(g_{\sigma^{\prime}} * \delta_{x} * \varphi_{\sigma}, \delta_{y}\right)
\end{aligned}
$$

In order to compute the above limit observe that the right hand side of 4.5 is given by $i \varepsilon \sum_{z \in G} g_{\sigma^{\prime}}(x z) \varphi_{\sigma}(z y)$. Since $g_{\sigma^{\prime}}$ a multiplicative function of $(x z)$ we can use this property providing that $|z| \geq|x|+2$. Hence we shall estimate $\sum_{|z| \geq|x|+|y|+3} g_{\sigma^{\prime}}(x z) \varphi_{\sigma}(z y)$.

4) Write $\frac{g_{\sigma+i 0}(x)-g_{\sigma-i 0}(x)}{g_{\sigma+i 0}(e)-g_{\sigma-i 0}(e)}$ for $\varphi_{\sigma}(x)$ and compute first

$\lim _{\varepsilon \rightarrow 0+} i \varepsilon\left(g_{\sigma^{\prime}} * \delta x * \varphi_{\sigma-\imath 0}, \delta_{y}\right)$. Define vectors $\underline{u}(x)=\left(u_{1}(x), \ldots, u_{q+1}(x)\right)$

$\underline{v}(x)=\left(v_{1}(x), \ldots, v_{q+1}(x)\right)$ as follows

$$
u_{j}(x) \sum_{t} g_{\sigma^{\prime}}\left(t x^{-1}\right) g_{\sigma-\imath 0}\left(t^{-1}\right)
$$

where the sum is taken over all elements $t \in G$ such that $|t|=|x|+1$ and the first letter of $t$ is not a power of $a_{j}$.

$$
v_{j}(x) \sum_{s} g_{\sigma-\imath 0}\left(s^{-1} y\right) g_{\sigma^{\prime}}(s)
$$

where the sum is taken over all $s$ in $G$ such that $|s|=|y|+1$ and the last letter of $s$ is not a power of $a_{\jmath}$. Recall that $g_{\sigma^{\prime}}(x)=\frac{1}{2 w\left(\sigma^{\prime}\right)} \cdot h_{\sigma^{\prime}}(x), g_{\sigma-i 0}(x)=$ $\frac{1}{2 w(\sigma-i 0)} h_{\sigma-10}(x)$ and define, for $n=1,2, \ldots(q+1)$ by $(q+1)$ matrices $A^{(n)}$ by the rule $A_{j, k}^{(n)}=\sum_{|t|=n} h_{\sigma^{\prime}}(t) h_{\sigma-i 0}(t)$ where the sum is taken over all elements $t$ of length $n$ such that the first letter is a power of $a_{j}$ and the last is a power of $a_{k}$. Define also a transition matrix $T$ letting

$$
T_{j, k}=\left\{\begin{array}{ll}
0 & \text { if } j=k \\
n \xi_{j}^{\prime} \xi_{j} & \text { if } j \neq k
\end{array} \quad j, k=1, \ldots, q+1\right.
$$


where $\xi_{j}^{\prime}=\xi_{j}\left(w\left(\sigma^{\prime}\right)\right)$ and $\xi_{j}=\xi_{j}(w(\sigma-i 0))$. Since $A^{(n+1)}=T A^{(n)}$, one can prove that

$$
\begin{aligned}
\left(g_{\sigma^{\prime}} * \delta_{x} * g_{\sigma-i 0}, \delta_{y}\right)= & \sum_{|t|<3+|x|+|y|} g_{\sigma^{\prime}}\left(t x^{-1}\right) g_{\sigma-t 0}\left(t^{-1} y\right) \\
& +\sum_{n=1}^{\infty} \underline{v}(y)\left(T^{n-1} A^{(1)}\right) \underline{u}(x) .
\end{aligned}
$$

5) In order to compute the limit in 4 , observe that the first term in the above equality remains bounded as $\varepsilon \rightarrow 0^{+}$, while the second term is nothing but $\underline{v}(y)(I-T)^{-1} A^{(1)} \underline{u}(x)$.

The caracteristic polynomial $P_{\varepsilon}(\alpha)$ of $T$ is given by

$$
P_{\varepsilon}(\alpha)=\left(\prod_{j=1}^{q+1}\left(\alpha+n \xi_{j}^{\prime} \xi_{j}\right)\right) \cdot\left(1-\sum_{j=1}^{q+1} \frac{n \xi_{j}^{\prime} \xi_{j}}{\alpha+n \xi_{j}^{\prime} \xi_{j}}\right) .
$$

Therefore, as $\varepsilon \rightarrow 0^{+} P_{\varepsilon}$ tends to a polynomial which has 1 as a simple root and this implies that, as $\varepsilon \rightarrow 0^{+}$, limit 4.6 is a product of the form $C(x) \cdot \varphi_{\sigma}(y)$.

As for the limit of $i \varepsilon\left(g_{\sigma+\imath \varepsilon} * \delta_{x} * g_{\sigma+\imath 0}, \delta_{y}\right)$ repeat the same reasoning, finding a matrix $T$ which, as $\varepsilon \rightarrow 0^{+}$, converges to a matrix which does not have the eigenvalue one. This implies that $\lim _{\varepsilon \rightarrow 0+} i \varepsilon\left(g_{\sigma+\imath \varepsilon} * \delta_{x} * g_{\sigma+i 0}, \delta_{y}\right)=0$.

\section{REFERENCES}

[A] K. Aomoto and Y. Kato, Green functions and spectra on free products of cyclic groups, Ann. Inst. Fourier, T. 38 (3988) Fasc. 1, 59-85.

[B] M. Bozeiko, Uniformely bounded representations of free groups, J. Reine Angew. Math., 377 (1987), 170-186

[C] P. Cartier, Harmonic analysis on trees, Proc. Symp. Pure Math., Amer. Math. Soc., 26 (1972), 419-424.

[C-FT] C. Cecchini and A. Figà-Talamanca, Projections of uniqueness for $L^{p}(G)$, Pacific J. Math., 51 (1974), 37-47.

[C-S] D.I. Cartwright and P.M. Soardi, Random walks on free products quotient and amalgams, Nagoya Math. J., 102 (1986), 163-180.

[D-S] N. Dunford and J.T. Schwartz, Linear operators, Interscience, New York, (1963).

[FT-S] A. Figà-Talamanca and T. Steger, Harmonic analysis for anisotropic random walks on homogeneous trees, to appear in Memories A.M.S.

[H] U. Haagerup, An example of a non-nuclear $C^{*}$-algebra which has the metric approximation property, lnvent. Math., 50 (1979), 279-293.

[K-S] G. Kuhn and T. Steger, Restrictions of the special representation of Aut $\left(\mathrm{Tree}_{3}\right)$ to two cocompact subgroups, to appear in Rocky Mountain J. Math.

[I-P] A. Iozzi and M. Picardello, Spherical functions on symmetrical graphs, Harmonic Analysis, Proceedings Cortona, Italy, Springer Lecture Notes in Math., 992 $344-387$. 
[L] E.C. Lance, $K$-theory for certain group $C^{*}$-algebras, Acta Matematica, 151 (1983), 209-230.

[ML] J.C. McLaughlin, Random walks and convolution operators, Doctoral Dissertation, New York Univ., (1986).

[M] W. Mlotkowsky, Positive definite radial functions on free products of groups, Boll. U.M.I., (7) 2B, (1988), 53-66.

[P] T. Pytlik, Radial functions on free groups and decompositionof the regular representation into irreducible components, J. Reine Angew. Math., 326 (1981), $124-135$.

[P-S] T. Pytlik and R. Szwarc, An analytic family of uniformly bounded representations of free groups, Acta Matematica, 157 (1986), 287-309.

$\left[\mathrm{T}_{1}\right] \quad$ A.R. Trenholme, Maximal abelian subalgebras of function algebras associated with free products, J. Funct. Anal., 79 (1988), 342-350.

$\left[\mathrm{T}_{2}\right]$ - Trenholme, A Green's function for non-homogeneous random walks on free products, Math. Z., 199 (1988), 425-441.

[S] T. Steger, Harmonic analysis for anisotropic random walks on homogeneous trees, Doctoral Dissertation, Washington Univ., St. Louis, (1985).

[V] D. Voiculescu, Addition of certain non-commuting random variables, J. Funct. Anal., 66 N.3, (1986), 323-346.

[W] W. Woess, Nearest neighbour random walks on free products of discrete groups, Boll. U.M.I., (6) 5B, (1986), 961-982.

Universita di Milano

Dipartimento di Matematica

"Federigo Enriques"

20133 Milano

Italia 